\title{
ON CERTAIN CONSTANTS ANALOGOUS TO FOURIER'S CONSTANTS.
}

BY DR. C. N. MOORE.

Througr the kindness of Professor Landau my attention has been called to the fact that Theorem I of the article of the above title which appeared in the BuLLETrN * may be obtained from a theorem due to Lerch $\dagger$ by means of a change of variable and a slight modification of the form of statement.

With regard to Theorem II of the same article I wish to state that the interval $a \leqq x \leqq b$ must be less than $2 \pi$ in order that the proof there given may hold. However, the theorem is still true when the interval in question is equal to $2 \pi$, and may be proved very simply from a theorem due to Fejér. The latter has shown + that if in an interval of length $2 \pi$ a function is finite save for a finite number of points, and is integrable, the Fourier development corresponding to that function is summable to the value of the function $\S$ at every point of that interval at which the function is continuous. Under the conditions of Theorem II the Fourier development corresponding to $\psi(x)$ in the interval $a \leqq x \leqq b$ has each of its terms equal to zero and is therefore summable to the value zero at every point of that interval. However, we know from Fejer's theorem that the development is summable to the value of the function $\psi(x)$ at every point of the interval $a \leqq x \leqq b$ at which this function is continuous and hence $\psi(x)$ is zero at every such point.

Cambridge, Mass., June, 1908.

* Bulletin, ser. 2, vol. 14 (1908), p. 368.

† Acta Mathematica, vol. 27 (1903), p. 345.

† Math. Annalen, vol. 58 (1903-04), p. 59.

\&. 1. e., the limit as $n$ becomes infinite of the arithmetic mean of $s_{1}, s_{2}, \cdots, s_{n}$, where $s_{m}$ represents the sum of the first $m$ terms of the series, exists and is equal to the value of the function. 